whether it causes less distress (which is not severe enough to be regarded as psychiatric caseness) and without remembering that the women's body image is preserved.

MARTIN S LeE

Department of Psychiatry,

Guy's Hospital, London SE

SIR,-We acknowledge that by virtue of their participation in a randomised study the patients assessed in the psychological arm of the Cancer Research Campaign's breast conservation trial might well be unrepresentative of patients with breast cancer in general.

The hypothesis suggested by Drs P C Milner and J P Nicholl (13 December, p 1568), however, is only one of many possibilities that might argue against the generalisability of our study. For these reasons we are currently conducting a "real world" psychological study, in which patients are in effect randomised to treatment by their referring GP. The psychiatric morbidity will be assessed in the patients of surgeons pursuing one of essentially three different treatment policies-that is, those offering primarily mastectomy, those offering primarily local excision, and those with no clear policy who feel that they should offer patients the choice of treatment. We hope that the findings of this multicentre study will be relevant to all the women in Great Britain currently undergoing treatment for breast cancer.

Finally, it is indeed ironic that one of us (MB) has contributed to the controversy concerning informed consent in randomised clinical trials, ${ }^{1}$ but we nevertheless feel that breast conservation represents a special case in which patients should be permitted to opt out if they express a clear preference for one or other treatment strategy for the primary complex. ${ }^{2}$

Michael Baum, LESLEY FALLOWFIELD

Department of Surgery,

King's College School of Medicine and Dentistry,

London SE5 9NU

1 Baum M. Do we need informed consent? Lancet 1986;ii:911-2.

2 Cancer Research Campaign Working Party in Breast Conserva-

tion. Informed consent: ethical, legal and medical implications for doctors and patients who participate in randomised clinical trials. BrMed J 1983;286:1117-21.

\section{Vegetarian diet in mild hypertension}

SIR,-Dr Barrie M Margetts and colleagues (6 December, $p$ 1468) show elegantly that a vegetarian diet, taken for six weeks, will reduce the systolic blood pressure by $5 \mathrm{~mm} \mathrm{Hg}$ on average. They state that the dietary component responsible for this change is a matter for speculation. The mechanism of the drop in pressure is even less clear and is not touched on. Our results on the blood rheology of vegetarians might stimulate discussion on this point.

We studied 48 voluntary vegetarians, measuring their blood and plasma viscosity, packed cell volume, red cell filterability, and aggregation. ${ }^{1}$ The results were compared with data from age and sex matched controls and showed a significant increase in blood fluidity in vegetarians. Baseline blood viscosity was lower in this group because of the decreased packed cell volume, but even when this was taken into account it was still significantly below the contro values. This can be explained by the fact that the plasma viscosity was also significantly lower than normal. By contrast, the rheological properties of red cells (filterability and aggregation) were no differen from those in controls. We also showed that the haemorrheological changes were more pronounced when the vegetarian diet was adhered to more strictly.

Finally, mean (SD) systolic blood pressure was lower in vegetarians: $109(7 \cdot 7) \mathrm{mm} \mathrm{Hg}$ in 28 ovolactovegetarians and $115(9 \cdot 8) \mathrm{mm} \mathrm{Hg}$ in 15 "semivegetarians," who consumed meat less than once a week.

The flow properties of blood represent the viscous component of the peripheral resistance. ${ }^{1}$ Hence the pressure needed to produce a given flow will be less when the viscosity is lower, with all other factors constant. Of course, this is a simplistic assumption, but our observation of "better than normal" blood rheology in vegetarians might, nevertheless, be linked to the authors' finding of the antihypertensive effect of a vegetarian diet in hypertensive patients.

E ERNST

A Matrai

L PIETSCH

Haemorrheological Research Laboratory,

Clinic for Physical Medicine,

University of Munich,

8000 Munich 2, FRG

1 Chien S, Dormandy J, Ernst E, Matrai A, eds. Clinical hemorheology. The Hague: Martinus Nijhoff, 1987.

\title{
Prescribing in pregnancy
}

SIR,-Messrs Martin J Whittle and Kevin P Hanretty (6 December, $p$ 1485) provide a figure for the incidence of central nervous system teratogenicity with valproate of $2-3 \%$, and later of about $2 \cdot 5 \%$, quoting the report by Lindhout and Schmidt. ${ }^{1}$ Because these figures are stated as if fact, and may be misquoted as such, it is important to consider the basis on which they are made.

An earlier report by Bjerkedal et al suggested that valproate probably caused spina bifida among about $1 \%$ of fetuses exposed to it in early pregnancy. ${ }^{2}$ The possibility of bias inherent in the study design was raised by Macrae. ${ }^{3}$

Lindhout and Schmidt reported the circulation of a questionnaire to 18 groups throughout the world carrying out prospective studies on the outcome of pregnancy in women with epilepsy: data from 13 groups were evaluated. ${ }^{2} A$ total of 2111 infants were exposed to any anticonvulsant drug and 12 had neural tube defects. The inference drawn was that the frequency of neural tube defect among infants exposed to valproate with or without other anticonvulsants was $1.5 \%(6 / 393)$, to valproate alone $2 \cdot 5 \%(3 / 120)$, and to other anticonvulsant drugs $0.35 \%(6 / 1718)$. However, examination of their data shows great discrepancies between the contributions of reported valproate exposures between the 13 centres-for example, Holland contributed $40 \%$ of the pregnancies in which exposure to valproate occurred, Plymouth and Fukushima 1\% each. This suggests different methods of collection of cases and pooling of potentially incompatible data.

The true incidence of neural tube defect with valproic acid or valproate is probably very low but has not been definitively determined. A spurious accuracy is given to estimates of incidence by quoting them to within half a per cent.

Sanofi UK Ltd,

Manchester M23 9NF

1 Lindhout D, Schmidt D. In-utero exposure to valproate and neural tube defects. Lancet 1986; :1392-3.

Bjerkedal T, Czeizel A, Goujard J, et al. Valproic acid and spin bifida. Lancet 1982;ii:1096.

3 MacRae KD. Sodium valproate and neural tube defects. Lancet 1982;ii:1283. E G Brown 